\title{
Modelling the Application Layer Protocols in IOT and Studying the Effective Transmission Mechanisms in Networks
}

\author{
Sridevi S. Taradi \\ Lecturer, IT dept \\ Vidyalankar \\ Polytechnic ,wadala
}

\begin{abstract}
The overwhelming and unprecedented need of research in the field of internet of things is forcing engineers, scientists and researchers to address communication and data distribution techniques in huge volume networks. MQTT and COAP are rapidly emerging as leading lightweight messaging protocols for gateway bridging logic to allow inter-standard communication. While billions of devices are connected over the internet it is necessary to study the packet transmission and evaluate the throughput of each transmission using traditional ALOHA and slotted ALOHA techniques[9].
\end{abstract}

\section{Keywords}

MQTT, COAP, LTN

\section{INTRODUCTION}

The buzz of "Internet of Things" began when Kevin Ashton coined the term to connect things via Internet through ubiquitous reality. The concept of creating a world where the devices could talk to each other, process information, take decisions requiring minimum human intervention came into picture. It has the capability to sense, analyse and visualize reality. IOT leads to the emergence of SMART environment which uses information and communication technologies like Wifi, Zigbee, RFID through devices which act as active participants that enable communication [4]. IOT is realized through sensors, actuators, gateway, cloud etc where sensors/actuators capture information; gateway forwards the information through internet and cloud is used to store and process information using vivid applications.

IOT can also be viewed as a three layer platform where machines talk at the interface level, communicate using internet at the middleware and apply semantic knowledge at the application level.

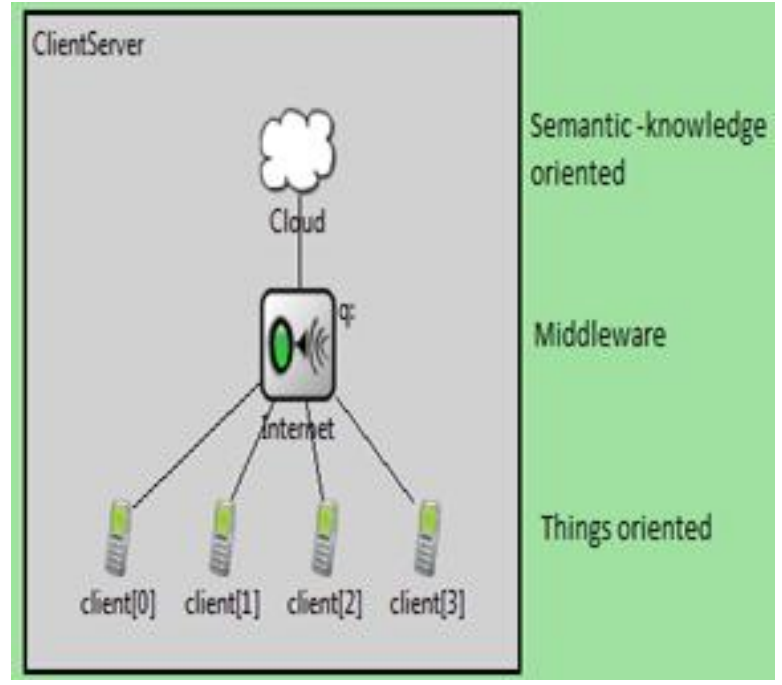

Fig 1: Diagrammatic representation of Internet of Things

2. APPLICATION LAYER PROTOCOLS IN IOT

$M Q T T$ and COAP

Message Queuing Telemetry Transport (MQTT) is the protocol which uses handshake mechanism to establish a connection followed by exchange of information between the devices where the provider acts as publisher and the requestor as the subscriber whereas Constrained Application protocol (COAP) is a UDP based protocol that uses a request-response mechanism to exchange message between the client and server devices [8]. 
Table 1. Comparison of MQTT and COAP

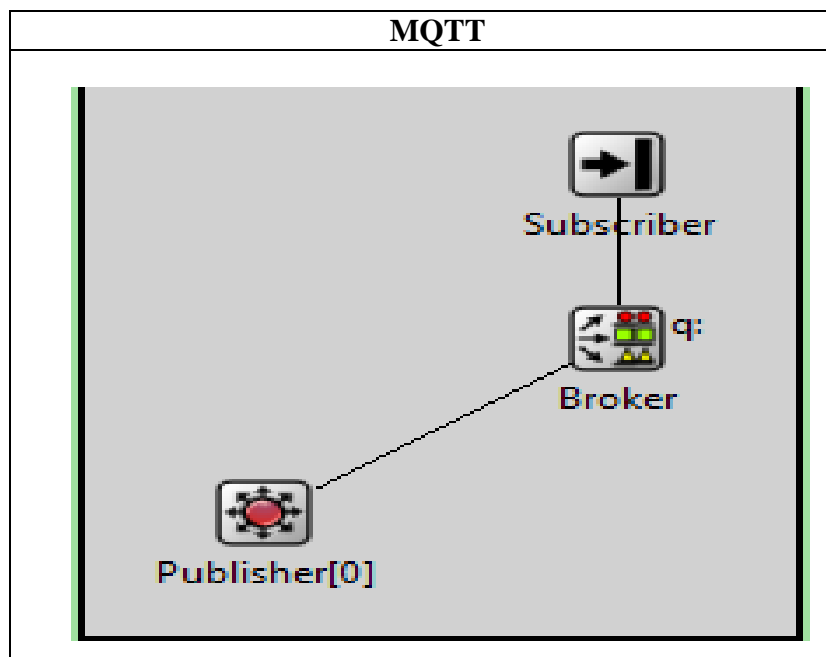

Fig 2: Communication Model in MQTT

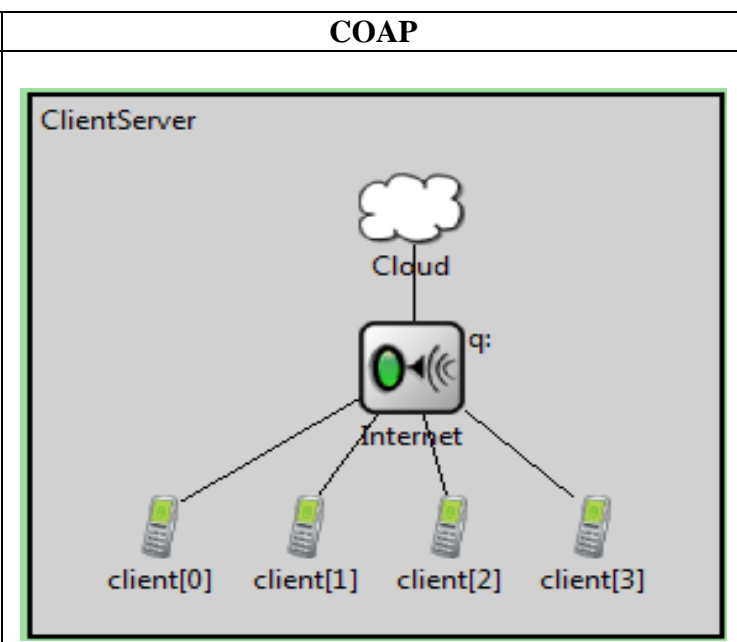

Fig 3: Communication Model in COAP

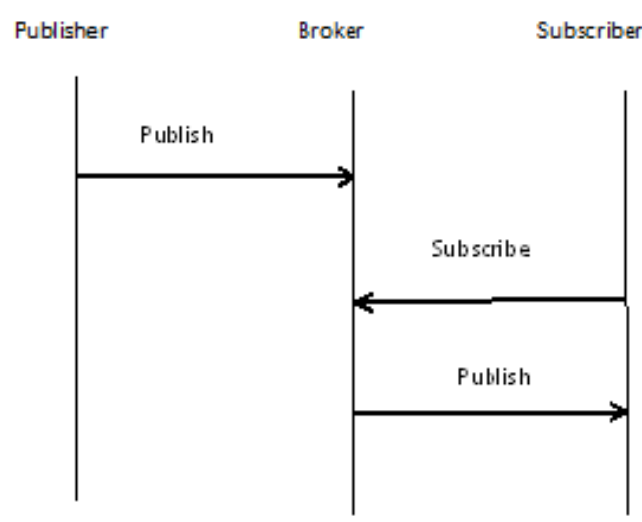

Fig 4: Publish-Subscribe method of communication
Fig 5: Request-Response method of communication

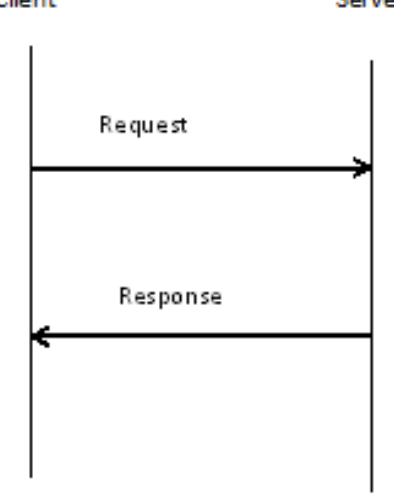

Table 2: Handshake mechanism in COAP[13]

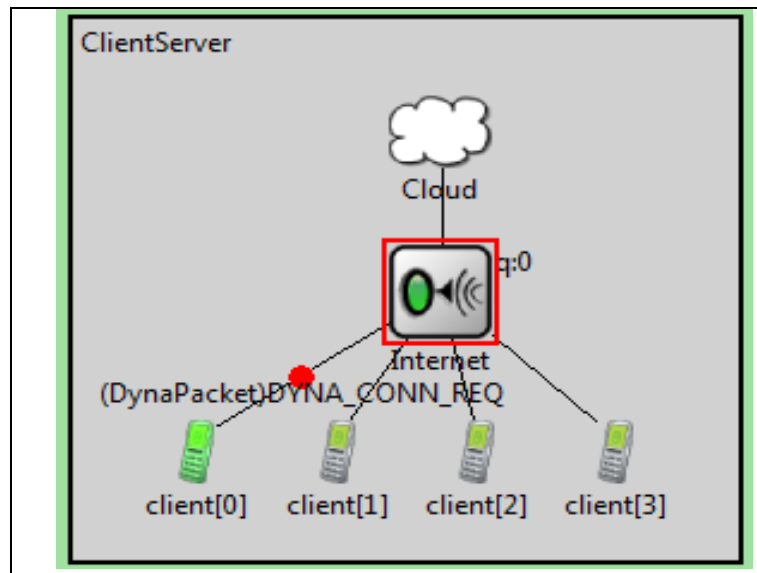

Fig 6: Client sends request

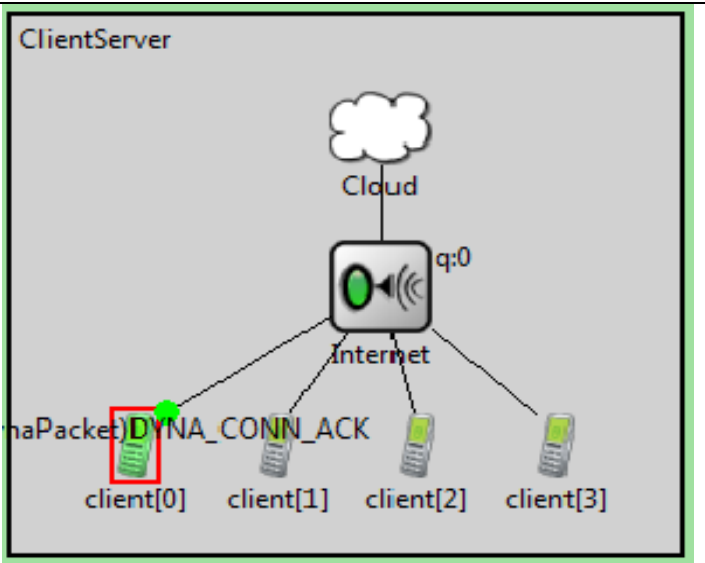

Fig 7: Server sends acknowledgement for request 


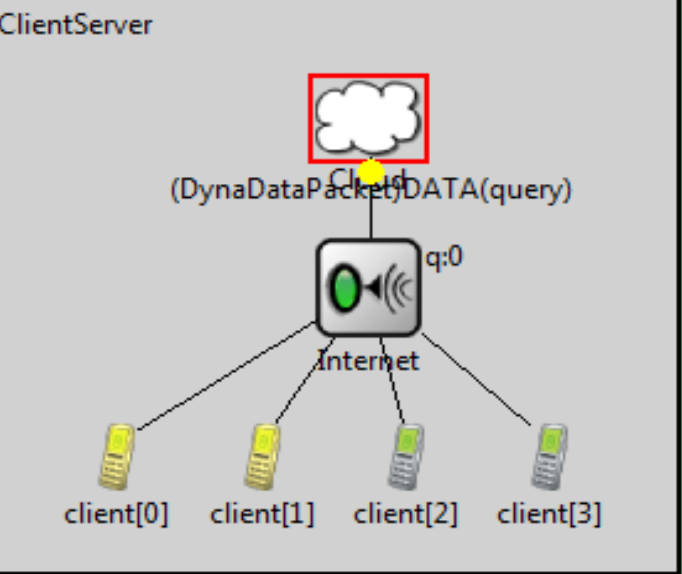

Fig 8: Client sends query

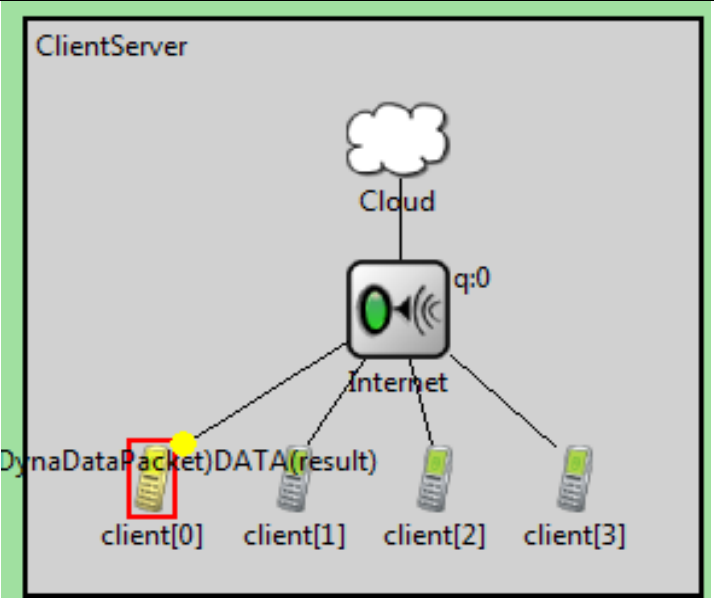

Fig 9: Server sends result of query
The handshake mechanism in COAP protocol can be explained by the table 2 where the devices send a connection request to the server which after being acknowledged sends the service request or query through the internet. The service request is processed by the applications running in the cloud which processes the information and make use of concept like software as a service or platform as a service to process the request.

\section{ALOHA AND SLOTTED ALOHA IN IOT}

The rise of cloud computing requires an accommodation of seamless integration of network access topologies demanding the advent of $5 \mathrm{G}$ in mobile communications which will deliver upto 5000 times the capacity of existing $3 \mathrm{G}$ and $4 \mathrm{G}$ technologies with a peak rate upto $100 \mathrm{Gbps}[1]$. Looking at the endless and deep rooted requirement of high speed, huge volume data transfer it is necessary to study packet transmission mechanisms.

\section{ALOHA:}

Assume that the same length packet frames are transmitted by stations following Poisson's distribution while transmission and that they do not generate while transmitting. Let $\mathrm{m}$ be the mean for Poisson's distribution for transmitting stations, probability of transmission attempt is given by,

\section{$\mathrm{P}($ transmission attempt $)=\mathrm{m} \mathrm{e}-\mathrm{m}$}

For just one station transmitting at a time, the probability of successful transmission is given by

$\mathrm{P}($ successful transmission $)=\mathrm{e}-\mathrm{m}$

While if there is another station attempting to transmit data during the same time frame, the probability of transmission attempt is given by,

$\mathrm{P}($ transmission attempt $)=\mathrm{P}($ successful transmission $)$ * $\mathrm{P}$ (transmission attempt)

$=\mathrm{e}-\mathrm{m} * \mathrm{~m}$ e-m

$=\mathrm{me}-2 \mathrm{~m}$

Therefore, the throughput of transmission is me- $2 \mathrm{~m}$.

\section{Slotted ALOHA:}

That in case of Slotted ALOHA assumes that all the packet transmission begin only at the start of the frame time inorder to avoid collision therefore there is no chance of another station starting the transmission[10][11][12].

Hence,

$\mathrm{P}($ transmission attempt $)=\mathrm{m}$ e- $\mathrm{m}$

Throughput $=$ me $-\mathrm{m}$

The IOT enabled devices will need to be ultra low latency i.e data transfer will take 1-10 milliseconds to reach from one designation to another compared to 40-60 seconds today[1]. With upto 50 billion devices to be connected through via Internet there arise a need to develop low throughput networks which will enable long range of around $40 \mathrm{~km}$ data transmission with minimal power consumption. Various standardization groups are working towards development of Low Throughput Networks(LTN) for Internet of Things networks dedicated to low throughput communications leading to the development of innovative technologies, applications and devices. These technologies also implements advanced signal processing that provides effective protection against interference [2][6].

As a result, LTN is particularly well suited for low throughput machine to machine communication where data volume is limited and low latency is not a strong requirement.

\section{CONCLUSION}

Enhancing the capability of IOT to dynamically configure the wireless network is the need of the hour to provide connections to the billions of connected objects. A great number of IOT driven objects need only low throughput connectivity, but they also require an efficient connection that is both cost effective and low energy-consuming. Standardization and collaboration of distinct IOT models, approaches, protocol structures, communication systems and applications is a must to establish a coherent universal network structure. Also, the incorporation of existing system designs, models, protocol layouts and techniques is essential to establish IOT at a residential level. Machine-to-Machine communications and the Internet of Things (IoT) involve the connection of potentially billions of connected objects which need only low throughput connectivity. A major and critical concern lies in avoiding collision during transmission and 
protection of catastrophic data, timely delivery even if collision happens because loss of single unit of data may interrupt operations of multiple devices talking amongst each other leading to error.

\section{REFERENCES}

[1] Dr. Ovidiu.V, Dr. Peter Friess , "IOT- From Research and Innovation to Market Deployment”,2014.

[2] http://www.etsi.org/images/files/ETSInewsletter/etsinew sletter_sep2016.pdf

[3] Yun Chao Hu, "Mobile Edge Computing (MEC)-a key technology towards 5G", September 2015.

[4] D. K. Klair, K Chin and R. Raad, "A Survey and Tutorial of RFID Anti-Collision Protocols", IEEE Communications Survey \& Tutorials, Volume: 12, Issue 3, pp. $400-421$

[5] Thomas F. La Porta, Gaia Maselli, and Chiara Petrioli, "Anti-collision protocols for single-reader rfid systems: temporal analysis and optimization", IEEE Transaction on Mobile Computing

[6] 5G Vision: The 5G Infrastructure Public Private Partnership: the next generation of communication networks and services. From The 5G Infrastructure Public Private Partnership:
[7] https://5gppp.eu/wpcontent/uploads/2015/02/5G-VisionBrochure-v1.pdf

[8] http://electronicdesign.com/iot/mqtt-and-coapunderlying-protocols-iot

[9] M. Zorzi, A. Gluhak, S. Lange, and A. Bassi, "From today's INTRANET of things to a future INTERNET of things: a wireless- and mobility-relatedview," IEEE Wireless Communications, vol. 17, no. 6, pp. 44-51, 2010.

[10] X. Wang, J. Kaniyil, Y. Onozato, and S. Noguchi, "Heterogeneous ALOHA networks: A sufficient condition for all equilibrium states to be stable," Computer networks and ISDN systems, vol. 22, no. 3, pp. 213-224, 1991

[11] Y. Jin and G. Kesidis, Equilibria of a noncooperative game for heterogeneous users of an ALOHA network," IEEE Commun. Lett.,vol. 6, no. 7, pp. 282-284, 2002

[12] M. S. Hefeida, T. Canli, and A. Khokhar , "CL-MAC: A cross-layer MAC protocol for heterogeneous wireless sensor networks," Ad Hoc Networks, vol. 11, no. 1, pp. 213-225, 2013.

[13] https://omnetpp.org/omnetpp/doc_details 